\title{
A Composite BRDF Model for Hazy Gloss
}

\author{
P. Barla ${ }^{1}$, R. Pacanowski ${ }^{2}$ and P. Vangorp ${ }^{3}$ \\ ${ }^{1}$ Inria \\ ${ }^{2}$ CNRS (LP2N) - IOGS - Université Bordeaux \\ ${ }^{3}$ Edge Hill University
}

\begin{abstract}
We introduce a bidirectional reflectance distribution function (BRDF) model for the rendering of materials that exhibit hazy reflections, whereby the specular reflections appear to be flanked by a surrounding halo. The focus of this work is on artistic control and ease of implementation for real-time and off-line rendering. We propose relying on a composite material based on a pair of arbitrary BRDF models; however, instead of controlling their physical parameters, we expose perceptual parameters inspired by visual experiments [VBF17]. Our main contribution then consists in a mapping from perceptual to physical parameters that ensures the resulting composite BRDF is valid in terms of reciprocity, positivity and energy conservation. The immediate benefit of our approach is to provide direct artistic control over both the intensity and extent of the haze effect, which is not only necessary for editing purposes, but also essential to vary haziness spatially over an object surface. Our solution is also simple to implement as it requires no new importance sampling strategy and relies on existing BRDF models. Such a simplicity is key to approximating the method for the editing of hazy gloss in real-time and for compositing.
\end{abstract}

CCS Concepts

-Computing methodologies $\rightarrow$ Reflectance modeling;

\section{Introduction and related work}

Real-world materials often exhibit reflections that may be described as "hazy", where more or less sharp specular reflections appear to be flanked by a halo. At the physical microscopic level, haziness may be due to various causes, such as partial polishing, diffraction effects, or multiple layers. As detailed in the following, two different approaches have been taken in the literature to design Bidirectional Reflectance Distribution Functions (BRDFs) that produce such hazy reflections: the aforementioned physical causes may be directly modeled, providing a predictive approach to material appearance; or a mathematical model with sufficient degrees of freedom (d-o-f) to reproduce haze effects may be provided. In this paper, we focus on the latter since artistic manipulation of hazy gloss is our central objective.

Physical models One way to produce hazy reflections is to make use of multi-layered BRDF models (e.g., [WW07, JdJM14]). They require the specification of many parameters such as the number of layers, their refractive indices, interface roughnesses and medium absorption and scattering properties. Hazy gloss may even be produced with a single interface thanks to diffraction effects. Existing models [CTL89, Sta99, LKYU12, HP17] are controlled by statistical properties of surface irregularities such as height roughness or auto-correlation distance, which have complex effects on the final appearance since they affect the BRDF in a wavelength-dependent manner. From a purely artistic point of view, these physicallyaccurate approaches provide too many d-o-f, while their parameters only indirectly control the final hazy appearance. In addition, they remain costly to apply in real-time, or even in production where rendering times directly affect budget costs.

Microfacet models The microfacet theory [CT82] has been widely adopted by the Computer Graphics community for its combination of physical plausibility and artistic ease-of-use. It relies on normal distribution functions, where different distributions lead to different specular highlight appearances. Until recently, the Beckmann distribution [BS63] was widely used; but the GGX distribution [TR75, WMLT07] gained favor over it due to its heavier tails. The main visual difference is that the GGX distribution produces slightly hazier reflections compared to Beckmann's; however, it does not provide any control over haze. Later work has introduced distributions with one additional d-o-f that controls distribution tails [LKYU12, BSH12, Bur12, RBMS17]. Unfortunately, none of them provides a satisfactory control over hazy gloss. The Shifted Gamma Distribution [BSH12], the peak of the distribution is strongly affected when attempting to modify its tails. The GTR [Bur12] and Student-t [RBMS17] distributions only permit achieving slightly hazier results than the GGX distribution. The $\mathrm{ABC}$ model [LKYU12] produces a halo of very large extent that cannot be controlled by the artist. 
Another simpler approach is to combine two or more distributions, which has oftentimes been deemed necessary for fitting BRDFs (e.g., [LFTG, NDM05]). Combination may also be performed manually for gaining more control over the shape of the specular term, as done by artists at Pixar or ImageWorks for instance $\left[\mathrm{HMC}^{*}\right]$. However, the manipulation of haze is only indirect in this case and requires trial and error. In particular, it is extremely difficult to create materials where haze varies spatially over a surface without affecting other material properties. When dealing with anisotropic materials, control becomes even more tedious since the number of parameters is increased.

Perception of haze Recent work in visual perception [VBF17] has shown that humans are visually sensitive to haziness in some specific configurations. The authors employ a specular BRDF based on two Ward BRDF components [War92], one of narrow extent (i.e., small roughness), the other of wide extent (i.e., large roughness). Their perceptual experiments show that haziness is a dimension of gloss that is distinct from contrast or distinctness of image [HH87, PFG00], and is more complex than the haze measurement retained by the ASTM [AST97]. Most importantly, none of the BRDF component parameters is able to account for perceived haziness: hence the perception of hazy gloss is not directly dependent on physical parameters. It is shown instead that a post-hoc decomposition of the BRDF into a specular core and a surrounding halo yields high correlation with subjective haziness ratings. Recent work (e.g., [SGM $\left.{ }^{*} 16\right]$ ) provides perceptual parameters to navigate the space of material appearance, yet none provides a direct control over haziness.

Our approach Throughout the paper, we focus on specular BRDFs and leave the diffuse term untouched. Our approach takes inspiration from the core/halo decomposition of Vangorp et al. [VBF17] to introduce a BRDF model that grants direct control over hazy effects in a physically-correct manner. We introduce material parameters that manipulate not only the haze magnitude but also its extent, which in practice exposes an additional d-o-f compared to distributions with controllable tails [LKYU12,BSH12, Bur12, RBMS17]. In particular, our method affects the distribution tails while leaving the specular core mostly unchanged (even with anisotropic materials), and permits producing a much more noticeable hazy gloss appearance compared to existing alternatives. This is achieved by using a sum of two arbitrary BRDFs having the same model (e.g., microfacet-based with GGX or Beckmann distribution), whose physically-based parameters are obtained by a mapping from our perceptually-based core and halo parameters, while guaranteeing reciprocity, positivity and energy conservation [NRH*77].

This simple approach has many benefits. It does not require any change in the rendering system, since only material parameters are modified; in particular, there is no need to introduce any new importance sampling strategy. It is also independent of the choice of distribution model, which makes it a viable solution for most rendering engines (such as those working with microfacet models); the method may even be implemented in the interface of a material editor. In spite of its simplicity, our method provides an efficient solution to a problem that would be hard to solve with a more complex single-component specular BRDF model.

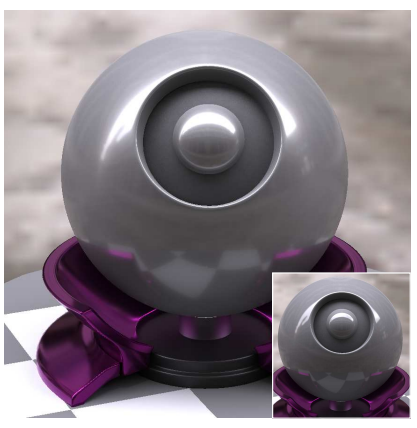

Probe A: dielectric

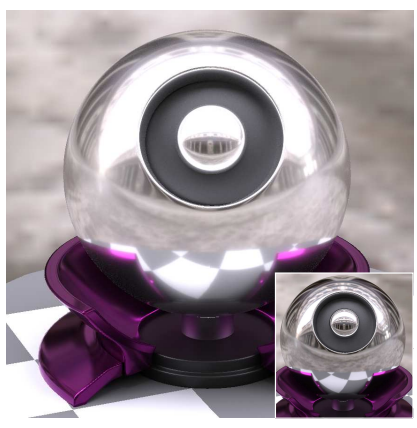

Probe B: conductor
Figure 1: A material probe rendered with our BRDF model in global illumination. The insets show the same object with haziness removed. Our method works with both dielectrics and conductors.

\section{Hazy gloss model}

We first introduce a physical BRDF model made of a sum of two specular components (Section 2.1), which we then reorganize into a sum of specular core and halo components following recent research in visual perception (Section 2.2). We next describe a parametrization that guarantees that material components stay within the space allowed by physical constraints (Section 2.3), before providing the full haze mapping from perceptual to physical parameters in algorithmic form (Section 2.4). Table 1 lists the symbols and notations used throughout this section.

\subsection{Physical BRDF model}

We consider a composite BRDF $f_{r}$ having a pair of components sharing the same Fresnel term:

$$
f_{r}\left(\omega_{i}, \omega_{o}\right)=\left((1-\beta) f_{n}\left(\omega_{i}, \omega_{o}\right)+\beta f_{w}\left(\omega_{i}, \omega_{o}\right)\right) F_{r}\left(\theta_{d}\right),
$$

where $\omega_{i}$ and $\omega_{o}$ are incoming and outgoing directions, $F_{r}$ is the Fresnel term, $\theta_{d}=\operatorname{acos}(\mathbf{h} \cdot \omega)$ is the difference angle with $\mathbf{h}=$ $\frac{\omega_{i}+\omega_{o}}{\left\|\omega_{i}+\omega_{o}\right\|}$ the halfway vector [Rus98], $f_{n}$ and $f_{w}$ are functions characterizing narrow and wide reflections respectively, and $\beta \in[0,1)$ linearly blends between the two functions. A common way to define $f_{n}$ and $f_{w}$ is through microfacet theory, in which case they model distributions of small and large roughness respectively ${ }^{\dagger}$. The mixture ratio $\beta$ then corresponds to the relative area occupied by the microfacets belonging to the distribution of large roughness. Whether the composite BRDF $f_{r}$ is physically-based obviously depends on whether $f_{n} F_{r}$ and $f_{w} F_{r}$ are themselves physicallybased, but also on their potential interactions through shadowing and masking (see Section 3).

The use of multiple components was first suggested in the seminal work of Cook and Torrance [CT82] on microfacet theory. It is nowadays routinely used in production $\left[\mathrm{HMC}^{*}\right]$, where such a combination of functions is primarily aimed at better shaping the

\footnotetext{
$\dagger$ Each function is of the form $f=\frac{D G}{4\left|\cos \theta_{i}\right|\left|\cos \theta_{o}\right|}$ where $D$ and $G$ are distribution and masking-shadowing terms respectively.
} 
Table 1: Table of symbols and notations

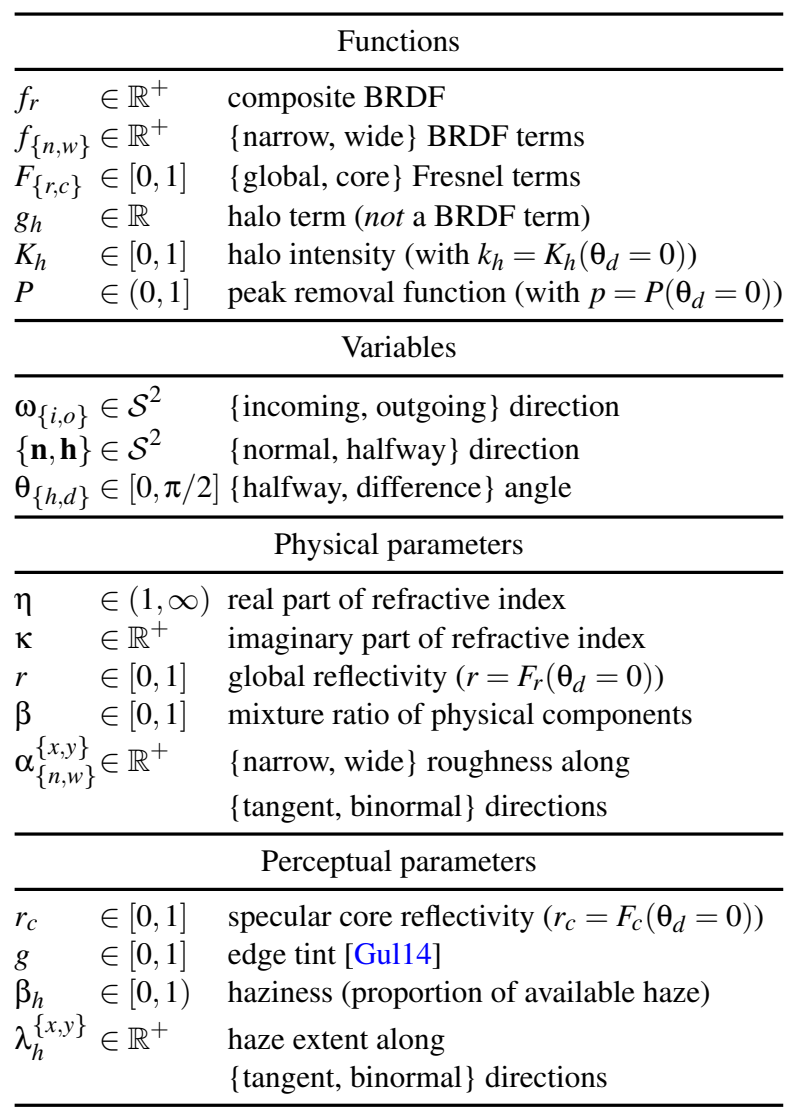

specular component. However, as shown in the left column of Figure 2, even though the model of Equation 1 yields a more complex specular BRDF, it does not provide an independent control over distribution tails. Indeed, when the intensity of the wide component is modified through $\beta$ (Figure $2 b$ ), not only the tails are modified, but the whole shape of the BRDF changes; in particular, the intensity in the mirror direction is affected. The same side-effect occurs when the roughness of the wide component is changed (Figure 2c). Having a direct and separate control over the peak and tails of the specular BRDF is not only desired to provide an artist-friendly handle on hazy gloss; it is also necessary if one wants to vary haziness across an object surface in a controllable manner.

\subsection{Perceptual decomposition}

Vangorp et al. [VBF17] have suggested that the perception of hazy gloss relies on a decomposition of the specular BRDF into a specular core and a surrounding halo. In particular, they show that the energy of their halo component is strongly correlated to subjective haziness ratings obtained through a perceptual experiment. However, they only propose a post-hoc analysis and do not discuss how to design a physically-based BRDF model providing a perceptually-relevant control over haze.

We take inspiration from their work to re-express the model of
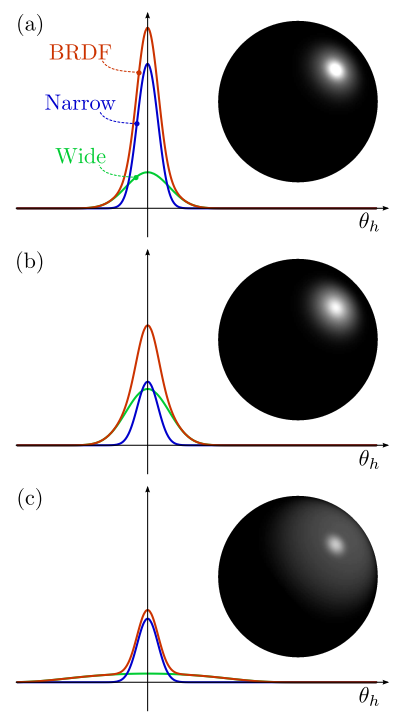

Physical BRDF
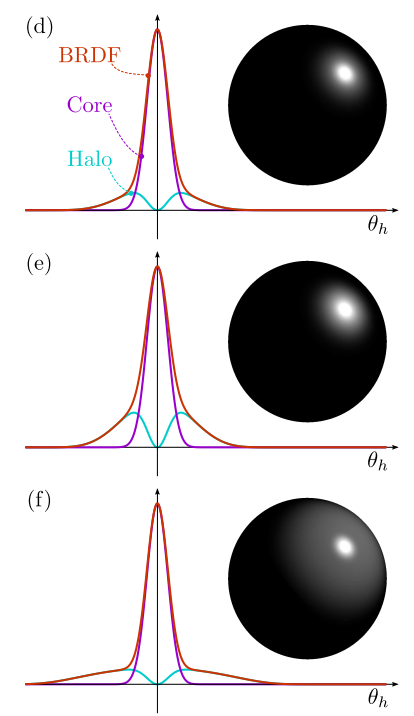

Perceptual decomposition

Figure 2: Comparison between the physical BRDF of Equation 1 and the perceptual decomposition of Equation 2. The narrow and wide components of the former are shown in blue and green respectively, while the specular core and surrounding halo of the latter are shown in magenta and cyan. Insets show renderings of a sphere with a directional light source. The two decompositions initially yield the same composite BRDF (in red), as shown in $(a, d)$. The $B R D F$ peak is altered when the parameters of the physical decomposition are modified to yield higher (b) or wider (c) tails. When the parameters of the perceptual decomposition are changed, the peak is not affected while the tails are modified as desired in $(e, f)$.

Equation 1 as the sum of a specular core and a surrounding halo:

$$
f_{r}\left(\omega_{i}, \omega_{o}\right)=F_{c}\left(\theta_{d}\right) f_{n}\left(\omega_{i}, \omega_{o}\right)+K_{h}\left(\theta_{d}\right) g_{h}\left(\omega_{i}, \omega_{o}\right) .
$$

More precisely, the specular core has the same shape $f_{n}$ as the narrow component of Equation 1, but is affected by a different Fresnel term $F_{c}$. The halo component is affected by an intensity $K_{h}$ and we propose to control its shape by $g_{h}=f_{w}-P f_{n}$, where $P \in[0,1]$ is computed so that the contribution of the halo in the specular direction is minimized. As illustrated in the right column of Figure 2, the purpose of this decomposition is to grant independent control over the peak and tails of the distribution.

Equation 2 is obtained from Equation 1 by adding $\beta F_{r} P f_{n}$ to the narrow component, and subtracting it from the wide component:

$$
\begin{aligned}
f_{r} & =(1-\beta) f_{n} F_{r}+\beta P f_{n} F_{r}+\beta f_{w} F_{r}-\beta P f_{n} F r \\
& =((1-\beta)+\beta P) F_{r} f_{n}+\beta F_{r}\left(f_{w}-P f_{n}\right) .
\end{aligned}
$$

By comparison with Equation 2 we obtain:

$$
\begin{aligned}
& F_{c}\left(\theta_{d}\right)=\left(1-\left(1-P\left(\theta_{d}\right)\right) \beta\right) F_{r}\left(\theta_{d}\right), \\
& K_{h}\left(\theta_{d}\right)=\beta F_{r}\left(\theta_{d}\right) .
\end{aligned}
$$

We emphasize that unlike $f_{n}$ or $f_{w}, g_{h}$ is not a valid BRDF function as it may become negative; neither is $K_{h}$ a valid Fresnel term. This is not problematic since Equation 2 is never directly evaluated 
but instead used for artistic control, after which its parameters are mapped back to physical parameters controlling Equation 1, which is evaluated. The only case where Equations 1 and 2 have the same form is when $\beta=0$ : the halo term then vanishes $\left(K_{h}=0\right)$ and the specular core becomes identical to the narrow component $\left(F_{c}=F_{r}\right)$.

An important consequence of the decomposition of Equation 2 is that for dielectric materials, the intensity of the specular core is uniquely determined by the $\theta_{d}=0$ configuration. Indeed, the Fresnel term $F_{c}$ is then uniquely determined by $r_{c}=F_{c}(0) .^{\ddagger}$ This means that Equation 3 only needs to be evaluated at $\theta_{d}=0$, hence knowing $p=P(0)$ is sufficient to yield a full bijection between Equations 1 and 2. Our goal is to make $g_{h}$ vanish in the specular direction, as shown in cyan in Figure $2 \mathrm{~d}-\mathrm{f}$. In the $\theta_{d}=0$ configuration, this translates to $g_{h}(\mathbf{n}, \mathbf{n})=0$, which is achieved by setting $p=\frac{f_{w}(\mathbf{n}, \mathbf{n})}{f_{n}(\mathbf{n}, \mathbf{n})}$. We may thus regard $p$ as the ratio of peak values between the wide and narrow components in the specular direction at normal incidence. The case of conductor materials may be treated similarly, as detailed in Section 2.4.

The exact analytic formula for the peak removal function $P$ actually depends on the choice of underlying BRDF model. As detailed in the supplementary document for the case of microfacet theory, when $\theta_{d}$ tends toward $\frac{\pi}{2}, P$ tends toward 1 . As a result, the separation between the specular core and halo is not perfect at grazing angles, which is necessary to obtain a valid BRDF. Fortunately, this has little visible impact on results since reflections are severely compressed close to object contours. We emphasize that the analysis of $P$ is not needed to implement the model, as we only require $p=P(0)$ to obtain a full bijection between Equations 1 and 2.

\subsection{Haze parametrization}

The specular core component of Equation 2 may be directly controlled by an artist, for instance by adjusting its reflectivity $r_{c}$ and its roughness in $f_{n}$. Our next goal is to find artistic parameters for controlling the halo component: its intensity $K_{h}$ and the extent of its shape $g_{h}$.

Haze intensity We want to provide a simple control over haze intensity $K_{h}$, while at the same time guaranteeing physical plausibility. One may suggest to let the artist directly control $\beta$ in Equation 4 . However, this would be equivalent to modifying the mixture weight in Equation 1, which does not provide an independent control over haziness as shown in Figure $2 b$. We must thus instead provide a control that modifies both $\beta$ and $F_{r}$ in Equation 4, while ensuring that the reciprocity, energy conservation and positivity constraints of the BRDF model are always ensured. The first of these constraints, reciprocity, is guaranteed provided that $f_{n}$ and $f_{w}$ are themselves reciprocal. The positivity constraint (denoted P) must apply to each of the narrow and wide components in Equation 1, yielding $(1-\beta) F_{r} \geq 0$ and $\beta F_{r} \geq 0$. The energy conservation constraint (denoted $\mathrm{E}$ ) is obtained by requiring that $F_{r} \leq 1 .{ }^{\S}$

\footnotetext{
$\ddagger$ The corresponding refractive index is given by $\eta_{c}=\frac{1+\sqrt{r_{c}}}{1-\sqrt{r_{c}}}$.

$\S$ This is equivalent to enforcing $F_{r} \in[0,1]$ and $\beta \in[0,1]$.
}

In order to write the $\mathrm{P}$ and $\mathrm{E}$ constraints in terms of $K_{h}$, we reexpress the global Fresnel term $F_{r}$ in terms of our decomposition:

$$
\begin{aligned}
F_{r}\left(\theta_{d}\right) & =(1-\beta) F_{r}\left(\theta_{d}\right)+\beta F_{r}\left(\theta_{d}\right), \\
& =F_{c}\left(\theta_{d}\right)-\beta F_{r}\left(\theta_{d}\right) P\left(\theta_{d}\right)+\beta F_{r}\left(\theta_{d}\right), \\
& =F_{c}\left(\theta_{d}\right)+\left(1-P\left(\theta_{d}\right)\right) K_{h}\left(\theta_{d}\right),
\end{aligned}
$$

where we have used Equation 3 in order to obtain the second line and Equation 4 for the third line.

We next observe that if the reflectivity $r=F_{r}(0)$ remains in the $[0,1]$ range, then Fresnel Equations ouput values $F_{r}\left(\theta_{d}\right) \in[0,1]$ as well for all $\theta_{d} \in\left[0, \frac{\pi}{2}\right]$. This means that we may safely restrict ourselves to the case where $\theta_{d}=0$ once again, and express the positivity and energy conservation constraints in terms of $k_{h}=K_{h}(0)$ :

$$
\mathrm{P}: 0 \leq k_{h} \leq \frac{r_{c}}{p} \quad \text { and } \quad \mathrm{E}: k_{h} \leq \frac{1-r_{c}}{1-p} .
$$

The new positivity constraint is obtained by applying Equation 3 to $(1-\beta) r \geq 0$, yielding $r_{c} \geq \beta r p$; then using Equation 4 to replace $\beta r$ by $k_{h}$. The new energy conservation constraint directly follows from Equation 5.

We visualize the constraints on $k_{h}$ as a function of $r_{c}$ using a pair of red lines in Figure 3-left. The two constraints intersect when $r_{c}=$ $p$ yielding $k_{h}=1$, which should be considered a limiting case since it amounts to having a single wide specular component. In order to control $k_{h}$, we propose to make use of a simple linear interpolation of $k_{h}$ from 0 to the physical bounds (black lines in Figure 3-left), which fully spans the space of valid BRDFs:

$$
k_{h}= \begin{cases}\beta_{h} \frac{r_{c}}{p} & \text { if } r_{c} \leq p \\ \beta_{h} \frac{1-r_{c}}{1-p} & \text { otherwise }\end{cases}
$$

where $\beta_{h} \in[0,1)$ is a haziness parameter that controls the proportion of available haze effect, given $r_{c}$ and $p$. We show the effect of $\beta_{h}$ on hazy gloss in Figure 3 for dielectric and conductor materials. Note that in the case of dielectrics, we use smaller values for $\beta_{h}$; indeed, higher values would yield a global reflectivity $r$ more typical of a conductor. As a rule of thumb, we usually set $\beta_{h} \in[0,0.1]$ for dielectrics, even though it may be increased for artistic purposes. We detail in the supplemental document the relationship between $\beta_{h}$ and the halo energy introduced by Vangorp et al. [VBF17] to explain subjective haziness ratings.

In some situations, the $C^{1}$ discontinuity of Equation 7 occurring at $r_{c}=p$ may be an issue. For instance, for smooth surface variations of the specular reflectivity $r_{c}$, the haze effect might exhibit visual discontinuities. We address this issue by replacing the piecewise linear form of Equation 7 with a quadratic rational Bézier curve (gray curves in Figure 3 ) that interpolates three control points: $\mathbf{p}_{0}=(0,0), \mathbf{p}_{1}=\left(p, \beta_{h}\right)$ and $\mathbf{p}_{2}=(1,0)$ with weights $\{1, w, 1\}$. It is guaranteed to remain in the $\left(\mathbf{p}_{0}, \mathbf{p}_{1}, \mathbf{p}_{2}\right)$ triangle. The additional parameter $w \in \mathbb{R}^{+}$controls the smoothness of the interpolation. Such a smooth interpolation comes at a price: only a subset of the space of parameters is then reachable. Equation 7 is obtained in the limit of $w \rightarrow \infty$. The formula for $k_{h}$ using rational Bézier interpolation is provided in the Appendix. 

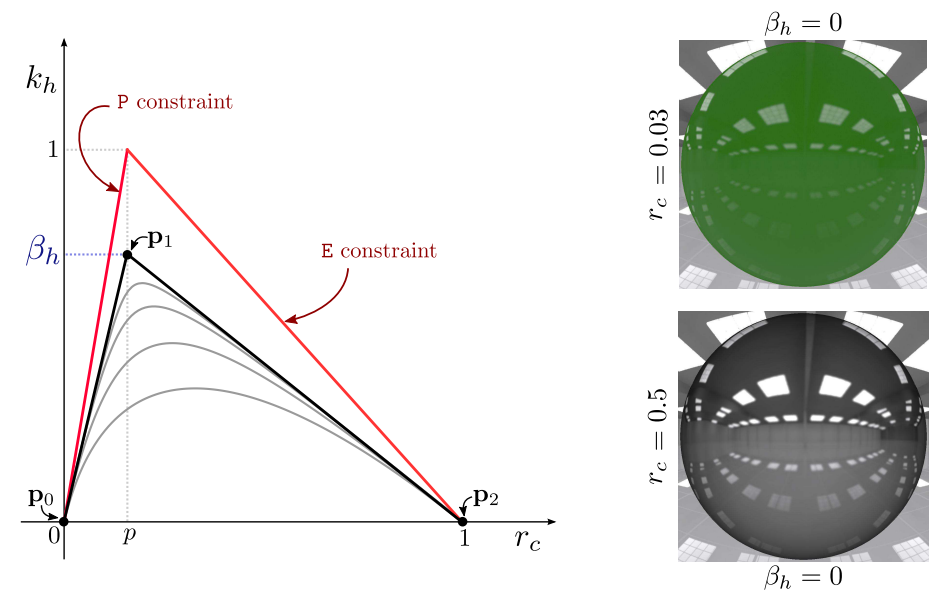
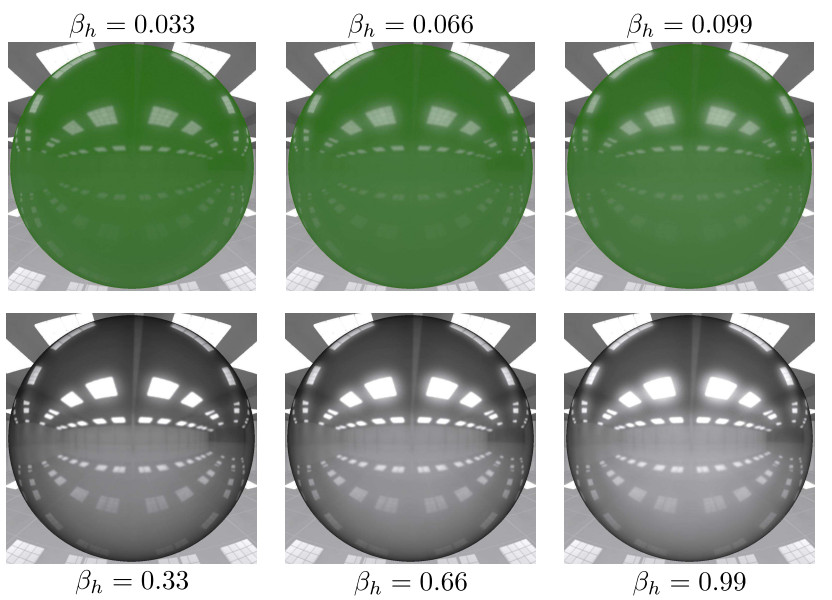

Figure 3: Left: the haze intensity $k_{h}$ at $\theta_{d}=0$ is given as a function of the core reflectivity $r_{c}$. The increasing (resp. decreasing) red line corresponds to the positivity $P$ (resp. energy conservation E) BRDF constraint. Valid values for $k_{h}$ (black lines) are obtained by linearly interpolating between 0 and the BRDF constraints, via a user-controlled parameter $\beta_{h} \in[0,1)$. In order to avoid the $C^{1}$ discontinuity at $r_{c}=p$, one may compute $k_{h}$ using a rational Bézier curve controlled by the points $\left\{\mathbf{p}_{0}, \mathbf{p}_{1}, \mathbf{p}_{2}\right\}$ and weights $\{1, w, 1\}$. Four such curves with weights $w \in\{1,2,4,8\}$ are shown in gray. Right: each row of rendered spheres shows materials with increasing haziness $\beta_{h}$ for a given core reflectivity $r_{c}$ typical of a dielectric on top, and of a conductor at bottom. We use $\alpha_{n}=0.01$ and $\lambda_{h}=7$ in both cases, and an additional greenish Lambertian term for the dielectric material.

Haze extent The angular extent of the halo component depends on $g_{h}$, and thus on the roughnesses of $f_{n}$ and $f_{w}$. Different BRDF models use different notations and formula for roughness; we will follow the most common notation and denote roughness by $\alpha$. For models that make use of different conventions (e.g., AshikhminShirley [AS00]), their parameter should be remapped to $\alpha$.

Let us first consider the isotropic case. By construction the roughness of the wide component $\alpha_{w}$ must be greater than the roughness of the narrow component $\alpha_{n}$. We choose to define the former using $\alpha_{w}=\alpha_{n}\left(1+\lambda_{h}\right)$, where $\lambda_{h} \in \mathbb{R}^{+}$controls the extent of the halo component in units of $\alpha_{n}$. This way, the extent of the halo component is relative to the extent of the specular core component. The configuration where $\lambda_{h}=0$ should be considered a limiting case, since the wide component then merges with the narrow one into a single-component BRDF. The first three columns of Figure 4 illustrate the effect of different values of $\lambda_{h}$ on the extent of the halo component.

For the general case of an anisotropic BRDF model, we denote by $\alpha_{n}^{x}$ and $\alpha_{n}^{y}$ the roughnesses of the narrow component in the tangential and binormal directions respectively, while $\alpha_{w}^{x}$ and $\alpha_{w}^{y}$ are the roughnesses of the wide component. We could have used equations similar to the isotropic case independently for each of the roughnesses along the tangential and binormal directions, but this would have had the effect of expressing haze extents in different units along different directions when $\alpha_{n}^{x} \neq \alpha_{n}^{y}$. We opt instead for the following definition:

$$
\left(\begin{array}{c}
\alpha_{w}^{x} \\
\alpha_{w}^{y}
\end{array}\right)=\left(\begin{array}{c}
\alpha_{n}^{x} \\
\alpha_{n}^{y}
\end{array}\right)+\left(\begin{array}{l}
\lambda_{h}^{x} \\
\lambda_{h}^{y}
\end{array}\right) \sqrt{\alpha_{n}^{x} \alpha_{n}^{y}},
$$

where $\lambda_{h}^{x} \in \mathbb{R}^{+}$and $\lambda_{h}^{y} \in \mathbb{R}^{+}$control the spread of the halo com- ponent in the same units along tangential and binormal directions respectively. When all components are isotropic, Equation 8 naturally reverts to the previously defined isotropic configuration. Our model also accommodates configurations where only one of the two components is isotropic, as shown in the last two columns of Figure 4. The supplemental document shows additional combinations of anisotropic roughnesses and haze extents.

\subsection{Haze mapping}

To recap, our approach essentially consists in a mapping from perceptual to physical parameters. It may actually be applied in the material editor of a rendering engine, either interactively when the artist is editing perceptual parameters, or as a batch process to convert textures storing variations of these parameters. The artist thus controls parameters of the specular core $\left(r_{c}, \alpha_{n}^{x}\right.$ and $\left.\alpha_{n}^{y}\right)$ and of the halo component $\left(\beta_{h}, \lambda_{h}^{x}\right.$ and $\left.\lambda_{h}^{y}\right)$. Next $k_{h}$ is computed using either Equation 7 or the smoothly-varying version using Equation 15 (see Appendix). The perceptual parameters are finally converted into the physical parameters of Equation 1, namely $r, \alpha_{w}^{x}, \alpha_{w}^{y}$, and $\beta=\frac{k_{h}}{r}$ (recall that $\alpha_{n}^{x}$ and $\alpha_{n}^{y}$ remain unchanged). Equation 1 is then evaluated during rendering.

For dielectrics, the global reflectivity $r$ might either be directly used to control Schlick's approximation [Sch94] to the Fresnel term, or converted to a refractive index $\eta=\frac{1+\sqrt{r}}{1-\sqrt{r}}$ to be used in the exact Fresnel equations.

For conductors, the refractive index is given by a complex number $\eta+i \kappa$. The additional $\mathrm{d}-\mathrm{o}-\mathrm{f}$ represented by the extinction coefficient $\kappa$ thus makes the mapping under-constrained. Ideally, we would like a solution that reverts to the case of dielectrics when 

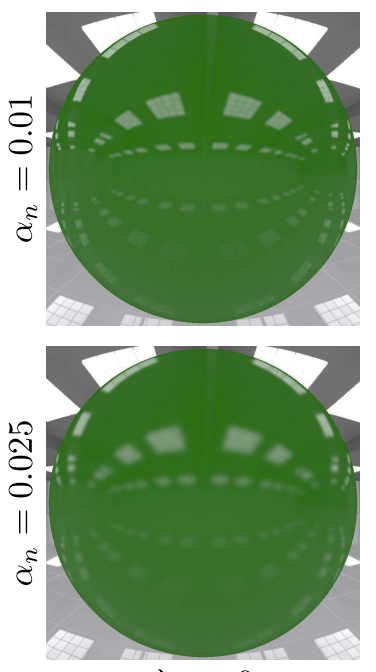

$\lambda_{h}=0$
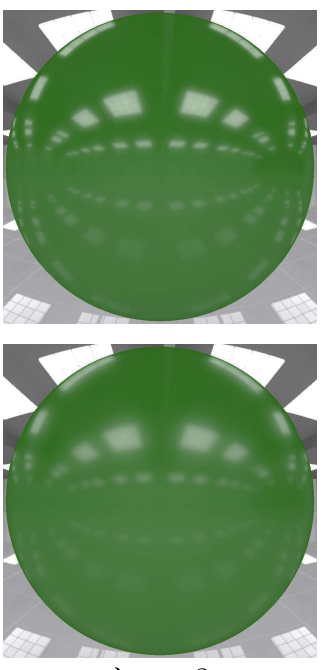

$\lambda_{h}=3$
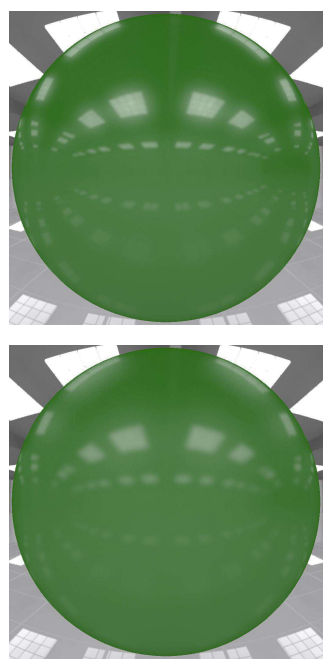

$\lambda_{h}=7$
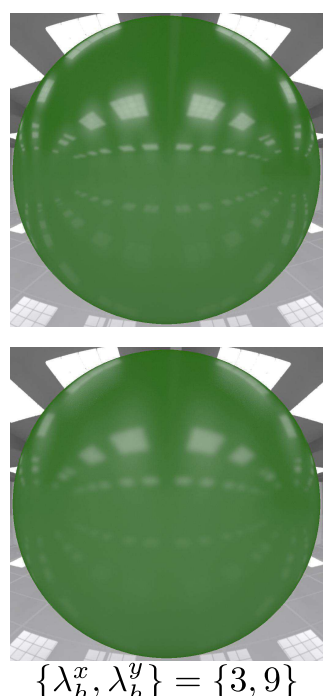

$\left\{\lambda_{h}^{x}, \lambda_{h}^{y}\right\}=\{3,9\}$
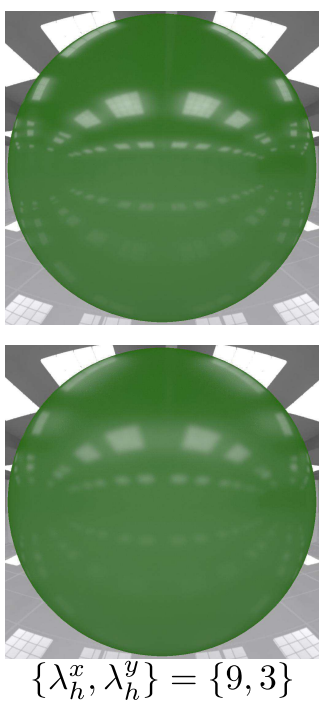

Figure 4: Each row shows materials with different narrow roughnesses $\alpha_{n}$ and varying haze extents $\lambda_{h}$ (with $r_{c}=0.03$ and $\beta_{h}=0.1$ fixed). The first column shows no haze; the next two columns show isotropic haze effects of different extents and the last two columns show anisotropic haze effects. Please zoom in to see haze effects.

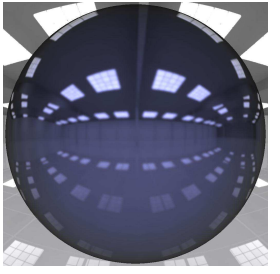

$\beta_{h}=0, \mathbf{g}=\mathbf{c}$

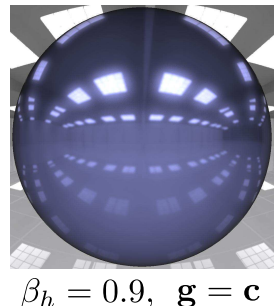

$\beta_{h}=0.9, \mathbf{g}=\mathbf{c}$

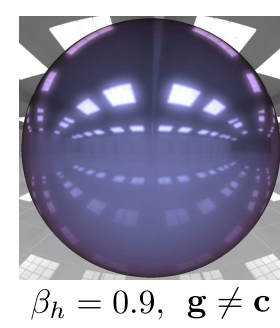

Figure 5: In the case of colored conductors (here $\hat{r}_{p}=0.5, \alpha_{n}=$ 0.01 and $\lambda_{h}=5.75$ ), we assign the same color to the core and halo components. Compared to dielectric materials, this leaves a d-o-f that is controlled by $\mathbf{g}$, an edge tint parameter [Gul14] that may be different from the reflectivity color $\mathbf{c}$ as seen in the rightmost image where reflected radiance takes on a purple tint near contours.

$\kappa=0$. We propose to rely on Gulbrandsen's artist-friendly mapping [Gul14] of reflectivity and edge-tint parameters into $\eta$ and $\kappa$. In practice, the artist provides a reflectivity $r_{c}$ and an edge-tint $g$ for the specular core. Our haze mapping converts $r_{c}$ to $r$ as before, but leaves $g$ unmodified. We finally apply Gulbrandsen's mapping to retrieve $\eta$ and $\kappa$ and use them in the Fresnel equations for conductors. Since $\kappa$ vanishes when $g$ vanishes, this approach generalizes the case of dielectrics as desired.

The method works for both achromatic and chromatic materials. In the latter case though, we must decide on the color of the halo component. We suggest that it should have the same chroma as the specular core component, which we achieve with the following approach. We first rewrite reflectivities as $\mathbf{r}$ and $\mathbf{r}_{c}$ to denote color vectors. We then define their chroma vector as $\mathbf{c}=\frac{\mathbf{r}}{\hat{r}}=\frac{\mathbf{r}_{c}}{\hat{r}_{c}}$, with $\hat{r}$ (resp. $\hat{r}_{c}$ ) the color coefficient of maximum intensity in $\mathbf{r}$ (resp. $\mathbf{r}_{c}$ ). We then use $\mathbf{r}=\hat{r} \mathbf{c}=\mathbf{r}_{c}+(1-p) \hat{k}_{h} \mathbf{c}$ in lieu of Equation 5, where $\hat{k}_{h}$ is obtained by replacing every occurrence of $r_{c}$ by $\hat{r}_{c}$ in
Equation 7. As shown in Figure 5, this gives the halo component the same color as the specular core, while leaving control over the edge tint color $\mathbf{g}$. This approach is compatible with a physicallybased BRDF, which requires $\beta$ to be a scalar: $\beta=\frac{\hat{k}_{h} \mathbf{c}}{\mathbf{r}}=\frac{\hat{k}_{h}}{\hat{r}}$.

As a summary, the general haze mapping is provided in the following Algorithm. In the next section, we explain how to instantiate it using various BRDF models.

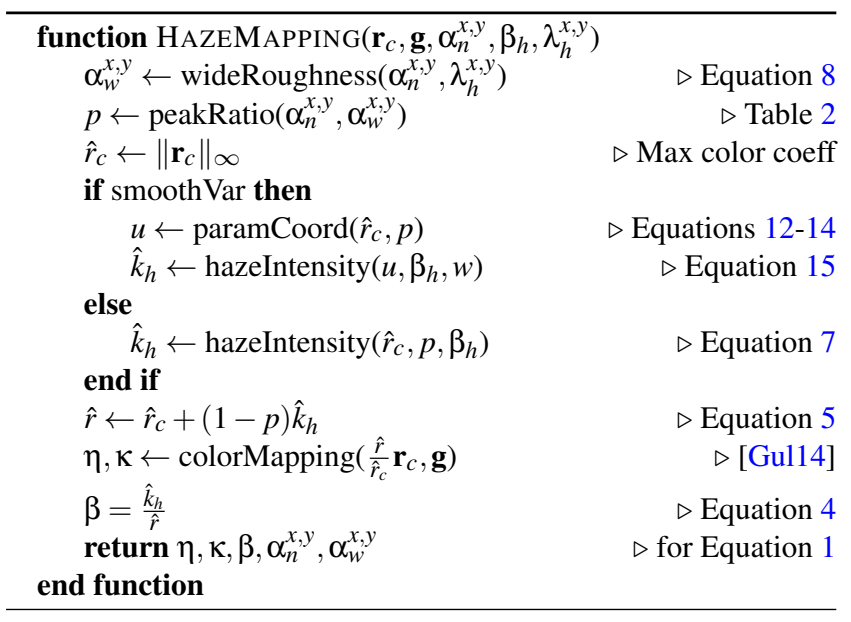

\section{Implementation details}

Choice of BRDF model Table 2 provides a list of common BRDF models that may be used to instanciate both $f_{n}$ and $f_{w}$, along with the corresponding formula for the peak ratio $p$ (see the supplementary document for derivations). The Blinn-Phong [Bli77] and Ashikhmin-Shirley [AS00] models use shininess $s_{\{n, w\}}$ instead of roughness $\alpha_{\{n, w\}}$, which yield somewhat complex for- 


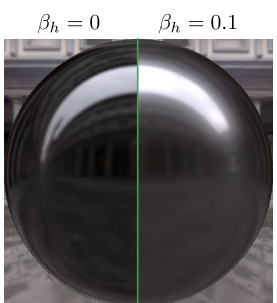

Ashikhmin-Shirley

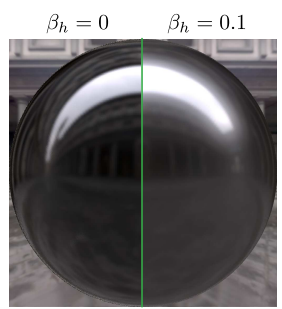

Ward (Beckmann)

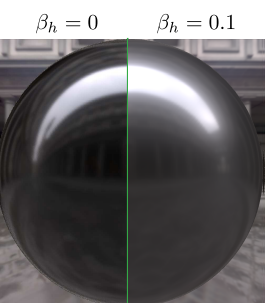

Walter et al. (GGX)
Figure 6: Our approach is modular in that it may be applied to a wide diversity of existing BRDF models. Here we show three such models, without and with haziness on each half. We fix $r_{c}=0.05$, $\alpha_{n}=0.02$ (or $s_{n}=5000$ for Ashikhmin-Shirley) and $\lambda_{h}=5$.

mula for $p$. In contrast, when using models involving either Beckmann [CT82, War92] or GGX [WMLT07] distributions, the peak ratio has a particularly concise formula. Note that our approach is compatible with models that have a coupled diffuse term designed to ensure energy conservation, such as Ashikhmin-Shirley [AS00].

Figure 6 shows the result of our approach when using the models of Ashikhmin and Shirley [AS00], Ward [War92] and Walter et al. [WMLT07]. Observe in particular how hazy gloss is consistently affected by modifying $\beta_{h}$ in the same manner irrespectively of the choice of underlying model. The Ward model is also used in Figures 3, 4, 5, 9 and 12; and the Walter et al. model in Figures 1, 11 and 13 . We have not included BRDF models with additional degrees of freedom [Bur12, LKYU12, BSH12, RBMS17] since our goal is to provide an alternative solution.

Our approach is also compatible with legacy BRDF models that do not make use of a Fresnel term (e.g., [Bli77, War92]). Equation 1 may then be written as $f_{r}=k_{n} f_{n}+k_{w} f_{w}$, where $k_{n}$ and $k_{w}$ are scalar coefficients for the narrow and wide components respectively, with $k_{n}+k_{w} \leq 1$ to preserve energy. This amounts to replacing the Fresnel term $F_{r}$ by the reflectivity $r$, and setting $k_{n}=(1-\beta) r$ and $k_{w}=\beta r$. The rest of our approach remains essentially unchanged: we only need to output $\mathbf{r}=\frac{\hat{r}}{\hat{r}_{c}} \mathbf{r}_{c}$ instead of the refractive indices $\eta$ and $\mathrm{K}$ in the haze mapping.

Table 2: Peak ratios for common BRDF models.

\begin{tabular}{lc}
\hline BRDF models for $f_{\{n, w\}}$ & peak ratios $p$ \\
\hline Blinn-Phong [Bli77] & $\frac{\left(s_{w}+2\right)\left(s_{w}+4\right)\left(2^{-s_{n} / 2}+s_{n}\right)}{\left(s_{n}+2\right)\left(s_{n}+4\right)\left(2^{-s_{w} / 2}+s_{w}\right)}$ \\
Ashikhmin-Shirley [AS00] & $\frac{\sqrt{\left(s_{w}^{x}+1\right)\left(s_{w}^{y}+1\right)}}{\sqrt{\left(s_{n}^{x}+1\right)\left(s_{n}^{y}+1\right)}}$ \\
Cook-Torrance [CT82] & $\frac{\alpha_{n}^{2}}{\alpha_{w}^{2}}$ \\
Ward [War92] & $\frac{\alpha_{n}^{x} \alpha_{n}^{y}}{\alpha_{w}^{x} \alpha_{w}^{y}}$ \\
Walter et al. [WMLT07] & $\frac{\alpha_{n}^{x} \alpha_{n}^{y}}{\alpha_{w}^{x} \alpha_{w}^{y}}$ \\
\hline
\end{tabular}

Compound masking-shadowing In the context of microfacet theory [CT82], Equation 1 should be interpreted as describing the

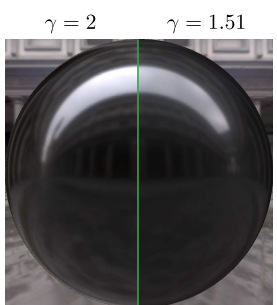

STD

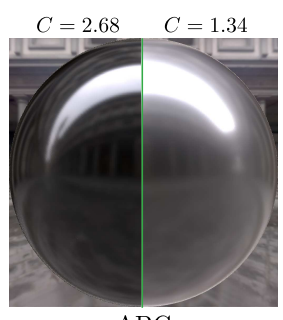

$\mathrm{ABC}$

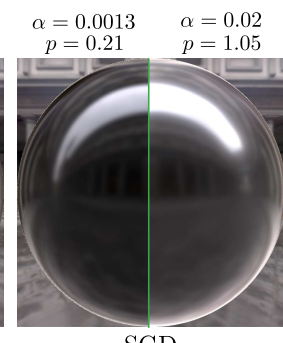

SGD
Figure 7: Recent BRDF models offer control over distribution tails thanks to an additional parameter; in each image, the left and right halves show renderings before and after parameter editing, as indicated on top. The Student-t distribution (STD) achieves slightly hazier but also significantly darker results. The ABC model produces strong haziness but with no control over its large extent. Our simplified version of the Shifted Gamma distribution (SGD) requires tedious manipulations of two parameters.

reflectance of a microsurface resulting from the mixture of two microfacet distributions. One may assume the mixture to be constructed of relatively large patches (on a micro-scale) of the component microsurfaces. The masking-shadowing effects across different patches may then be considered as negligible. In contrast, if the two distributions are intertwined, then it becomes necessary to consider a compound masking-shadowing term.

For most microfacet-based models, the masking-shadowing term does not depend on roughness parameters; the compound term is then identical for narrow and wide components. An exception is Smith's model [Smi67], which offers the most physically-realistic option [Hei14]. In its separable form, it is given by $G_{2}\left(\omega_{i}, \omega_{o}\right)=$ $G_{1}\left(\omega_{i}, \mathbf{h}\right) G_{1}\left(\omega_{o}, \mathbf{h}\right)$, with $G_{1}(\omega, \mathbf{h})=\frac{\chi^{+}(\omega \cdot \mathbf{h})}{1+\Lambda(\omega)}$ where $\chi^{+}(x)$ equals 1 if $x$ is positive and 0 otherwise. In our case, $\Lambda(\omega)=(1-$ $\beta) \Lambda_{n}(\omega)+\beta \Lambda_{w}(\omega)$, where $\Lambda_{n}$ and $\Lambda_{w}$ correspond to integrals over slopes of the narrow and wide distributions respectively.

The use of a large-patch or intertwined mixture of distributions is a matter of choice, and our approach is independent of this choice. Indeed, we rely on the peak ratio $p$ in our haze mapping, which only considers the configuration $\left(\theta_{h}, \theta_{d}\right)=(0,0)$, in which case the masking-shadowing term is equal to 1 (all microfacets are visible at normal incidence). We discuss the visual impact of using a compound masking-shadowing term in the supplementary document.

\section{Results}

We first compare our compound BRDF to existing models in Section 4.1. More complex rendering results - including spatial material variations, global illumination and transmission - are presented in Section 4.2. We also consider approximations that permit adapting our approach to the constraints of real-time or compositing applications in Section 4.3.

\subsection{Comparisons}

There is no easy way to compare different BRDF models since they rely on different material parameters; we thus resort to a quali- 

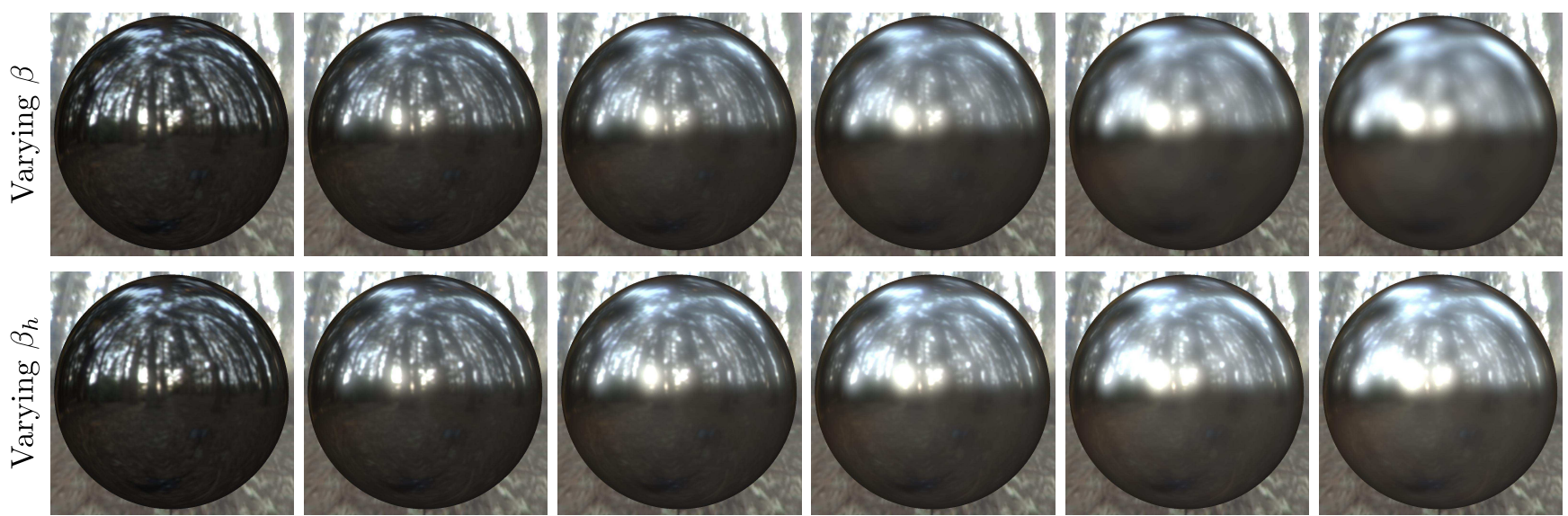

Figure 8: We compare variations of the mixture weight $\beta$ in the physical model (top row) with variations of haziness $\beta_{h}$ in the perceptual model (bottom row). Both parameters are uniformly sampled in the $[0,0.95]$ range; other parameters are chosen to get identical images in the left column $\left(\alpha_{n}=0.012, r=r_{c}=0.5, \alpha_{w}=0.088\right.$ or equivalently $\left.\lambda_{h}=6.4\right)$. The visual differences are mostly apparent in the three right-most images: increasing $\beta$ decreases the intensity of sharp reflections; increasing $\beta_{h}$ only affects haziness.

tative evaluation. To support this comparison, we provide GLSL shaders to be used in BRDF Explorer [Dis11] for each of the considered models, and encourage the reader to edit their parameters and compare their expressivity and ease of use. We have used these shaders in Figure 7 and attempted to find sets of parameters for the STD [RBMS17], ABC [LKYU12] and SGD [BSH12] models that would yield similar visual appearances before editing (left halves).

We start with the STD model [RBMS17] in the left of Figure 7. It is similar to the GTR distribution [Bur12], but provides an analytical masking-shadowing term. Moreover, it generalizes both the GGX and Beckmann distributions through a single parameter $\gamma$ that controls distribution tails. In particular, the left half of the figure is made identical to the left half of Figure $6(\mathrm{GGX})$ by setting $\gamma=2$. As shown in the right half of the figure, setting $\gamma$ to its minimal feasible value produces a slightly hazier but also significantly darker appearance. This limitation has been acknowledged by the authors.

The ABC model is shown in the middle of Figure 7. The formulation of Löw et al. [LKYU12] is inspired by diffraction-based models [CTL89] but rewritten as a microfacet-based BRDF. Even though their masking-shadowing term is not physically-based, the model retains interest as it permits control of distribution tails through the $C$ parameter. As shown in the right half of the figure, the hazy appearance is much stronger when increasing $C$, but at the same time it exhibits a very large extent. Since no other material parameter is provided to adjust haze extent, the model remains limited for artistic editing.

The SGD model [BSH12] is shown at right in Figure 7. It has originally been introduced for the fitting of BRDF data; here we use a simplified version of the model for the purpose of parameter editing (see the supplemental document for details). Unfortunately, as opposed to the two previously discussed models, a desired hazy appearance requires the modification of two parameters instead of one. This not only implies significant trial and error, but also precludes the use of the model for spatially-varying haziness. The model also shares the limitation of the ABC model in that it does not provide control over haze extent, even though its extent is less pronounced.

In contrast, our composite model provides a direct control over both haziness and haze extent through dedicated parameters (i.e., $\beta_{h}$ and $\lambda_{h}$ ) while retaining physical validity; this may be seen by comparing Figures 6 and 7. One might wonder whether the mixture parameter $\beta$ of the physical model of Equation 1 could be used to control haziness as well. As shown in Figure 8, while a linear variation of $\beta_{h}$ produces a consistent change in haziness throughout variations, the same linear variation of $\beta$ makes the material look hazy at low values, but makes it look mostly very rough at higher values. We again invite the reader to compare the two BRDF models using the provided shaders for BRDFExplorer (we also include executables for ease of use, as well as video captures).

\subsection{Rendering}

As with any other BRDF model, rendering is achieved through the reflected radiance equation [Kaj86]:

$$
L_{r}\left(\mathbf{x}, \omega_{o}\right)=\int_{\Omega} f_{r}\left(\mathbf{x}, \omega_{i}, \omega_{o}\right) L_{i}\left(\mathbf{x}, \omega_{i}\right) \omega_{i} \cdot \mathbf{n} d \omega_{i},
$$

with $L_{r}$ and $L_{i}$ the reflected and incoming radiances, $\mathbf{x}$ a surface point and $\Omega$ the hemisphere centered around the normal $\mathbf{n}$. In our case, Equation 9 takes the form $L_{r}=(1-\beta) L_{n}+\beta L_{w}$ where $L_{n}$ and $L_{w}$ are obtained by replacing $f_{r}$ by $f_{n} F_{r}$ and $f_{w} F_{r}$ respectively. As a result, there is no need for any new importance sampling strategy: we sample one randomly chosen component with probability $1-\beta$ for $L_{n}$ and $\beta$ for $L_{w}$.

A key benefit of our perceptual decomposition is that it grants spatial variations of both haziness $\beta_{h}$ and haze extent $\lambda_{h}$ independently of the specular core. This is shown in Figure 9, where we compare variations of the physical and perceptual parameters: only in the latter case does hazy gloss vary in a consistent and controllable way. Figure 10 shows our model applied with more complex spatial variations. We also show in the supplemental document how our model behaves with variations of the core reflectivity $r_{c}$. 

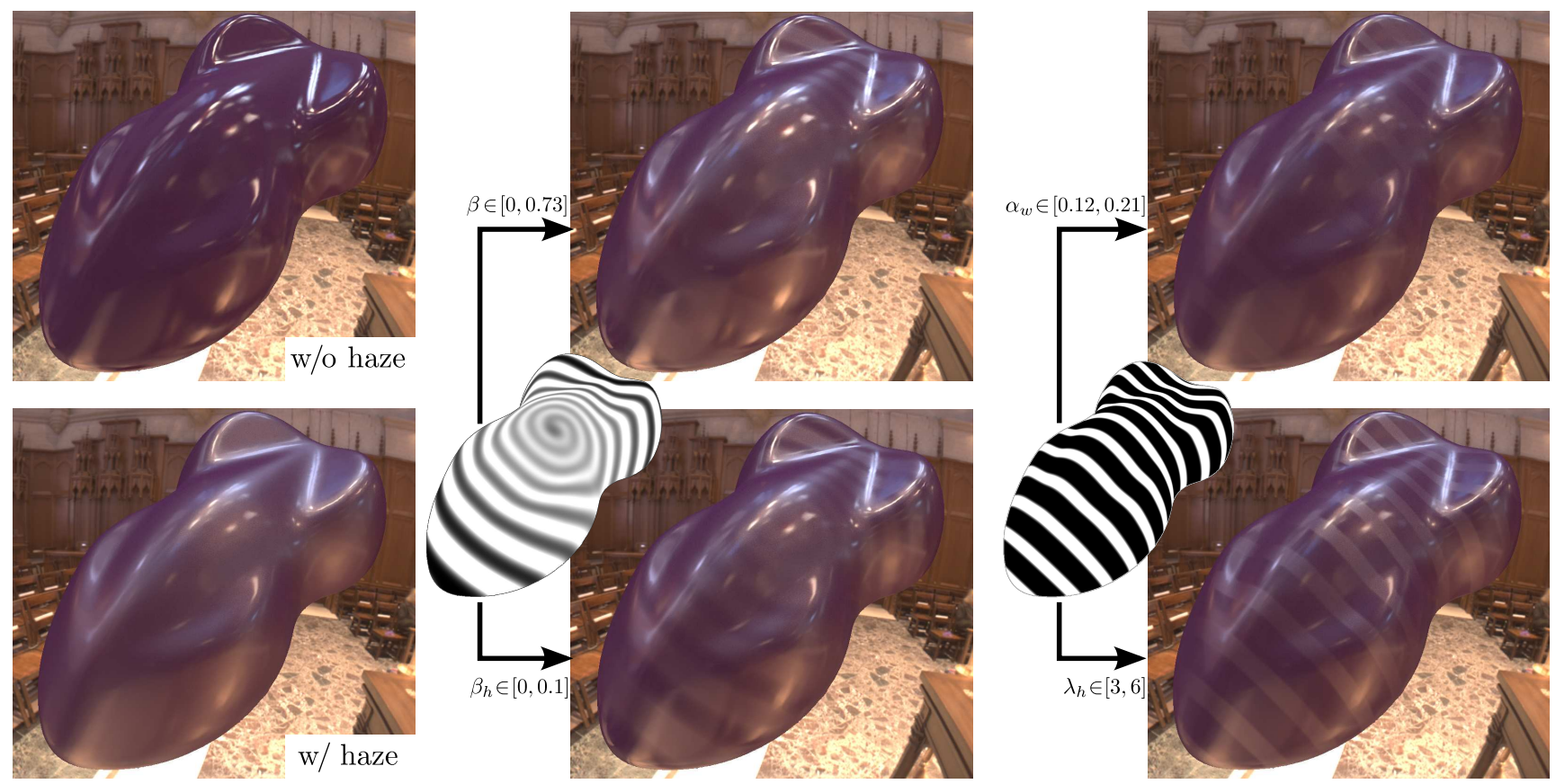

Figure 9: Left: the Speedshape model rendered with and without haze (we use $r_{c}=0.04, \alpha_{n}=0.03, \beta_{h}=0.1$ and $\lambda_{h}=5$ and a purple Lambertian base). Middle: when spatial variations are applied to haziness $\beta_{h}$ (bottom), the spiral pattern appears clearly, as opposed to when we vary $\beta$ (top). Right: variations of haze extent $\lambda_{h}$ (bottom) are much more noticeable compared to variations of wide roughness $\alpha_{w}$ (top). The physical parameter ranges for $\beta$ and $\alpha_{w}$ are computed using our haze mapping for the sake of comparison.
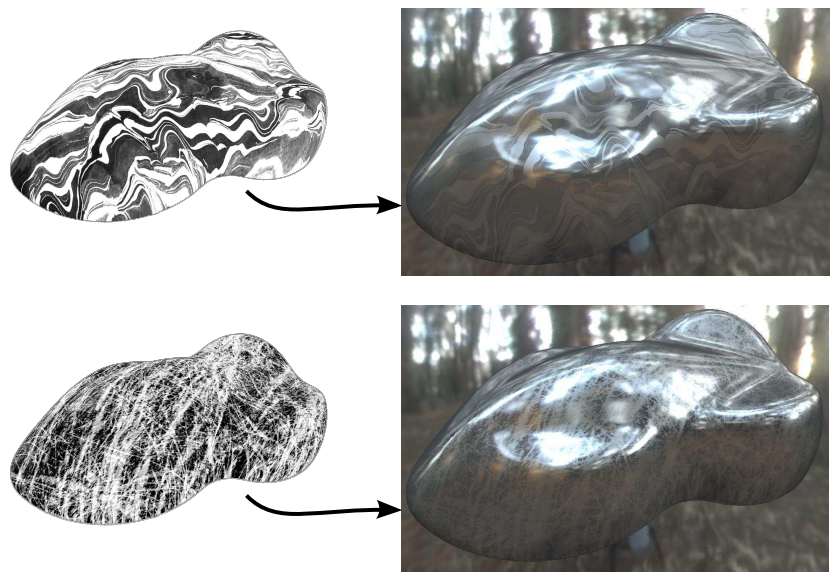

Figure 10: Detailed variations of haziness $\beta_{h} \in[0,0.99]$. Other parameters are kept fixed at $r_{c}=0.5, g=0, \alpha_{n}=0.026$ and $\lambda_{h}=5$.

Table 3: List of parameters used in Figures 1 and 11

\begin{tabular}{|l|c|c|c|c|c|}
\hline & $r_{p}$ & $g$ & $\alpha_{n}$ & $\beta_{h}$ & $\lambda_{h}^{\{x, y\}}$ \\
\hline Probe A & 0.02 & 0 & 0.01 & 0.1 & 5 \\
Probe B & 0.5 & 1.0 & 0.01 & 0.99 & 5 \\
Fertility & {$[0.5,0.28,0.1]$} & {$[0.8,0.06,0.03]$} & 0.03 & 0.99 & 5 \\
Vase & 0.05 & 0.0 & 0.01 & 0.15 & 7 \\
Teapot & {$[0.5,0.12,0.12]$} & {$[0,0.85,1]$} & 0.01 & 0.99 & $\{10,1\}$ \\
\hline
\end{tabular}

Figure 1 demonstrates the use of our model in global illumination rendering. Each material probe is shown with and without haze on the spherical part (other parts are left unchanged). Observe how our method preserves the intensity of sharp specular reflections for either dielectrics, achromatic conductors or colored conductors. Figure 11 shows a more complex scene that showcases various hazy materials: dielectrics and conductors, isotropic and anisotropic. The material parameters are listed in Table 3. Our model is not limited to opaque materials as shown in the left of Figure 13. Rendering with Bidirectional Transmittance Distribution Functions (BTDF) actually requires no modification to our approach — another advantage of relying on existing material models through a simple haze mapping. We simply use the same physical parameters (mixture ratio $\beta$, refractive index $\eta+i \kappa$ and roughnesses $\alpha_{\{n, w\}}^{\{x, y\}}$ ) in a two-component BTDF of a form similar to Equation 1.

\subsection{Approximations}

In video games, the need for balancing high performance, low memory consumption and realistic appearance has led to the use of local lighting environments pre-filtered for a range of material parameters [KVHS00]. In order to avoid precomputing and storing high-dimensional lookup tables, recent work (e.g., [Kar13]) has proposed to re-organize Schlick's approximation [Sch94] of the Fresnel term. Omitting function variables for clarity, the Fresnel term is then re-expressed as:

$$
F_{r}=(1-\Delta) r+\Delta, \quad \text { with } \quad \Delta=\left(1-\cos \left(\theta_{d}\right)\right)^{5} .
$$



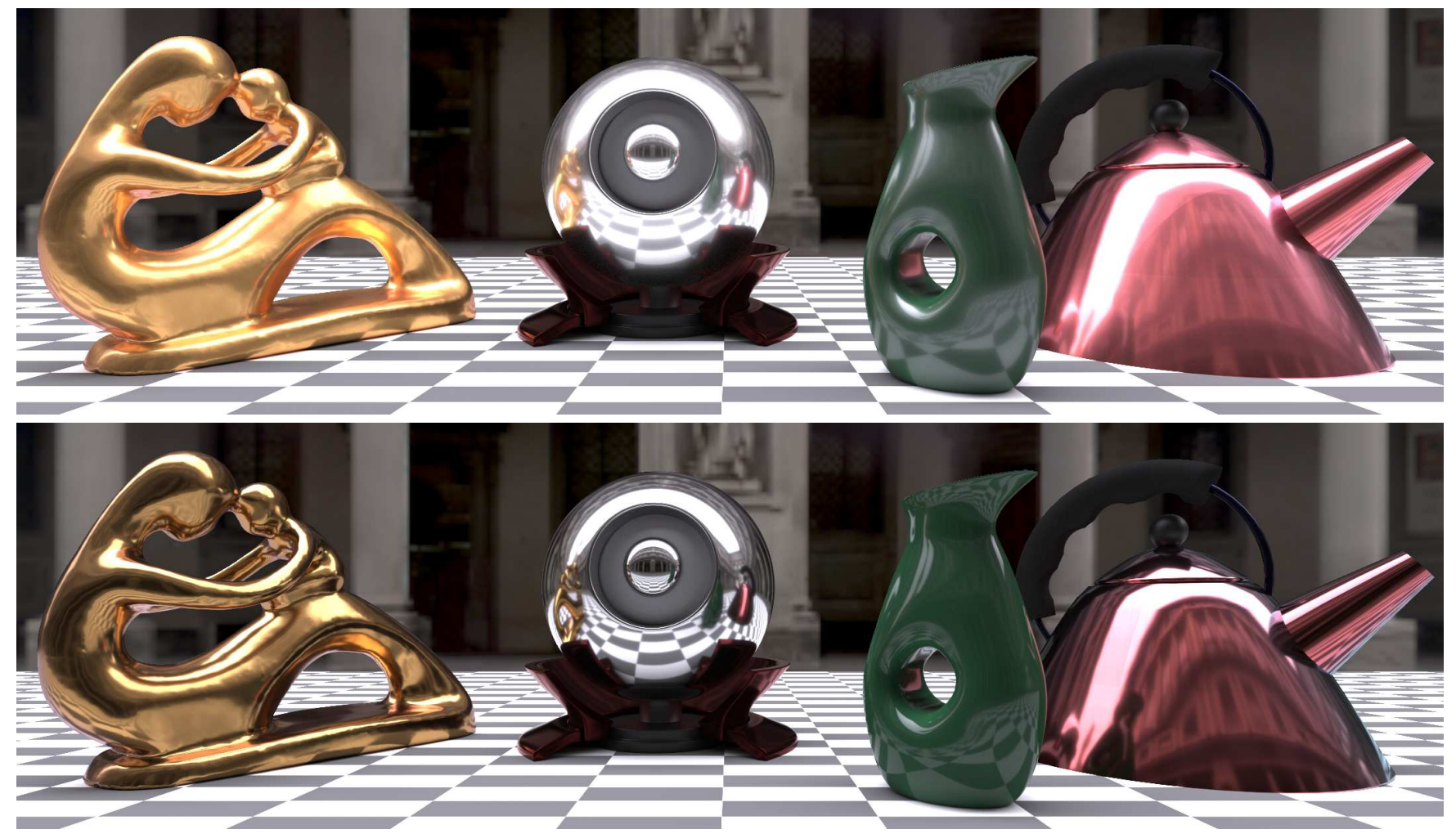

Figure 11: A 3D scene rendered with path tracing using hazy (top) and haze-free (bottom) materials. From left to right: the Fert il it $y$ statue is made of a colored conductor using a wide haze extent and a pinkish edge tint; the Probe B object uses the achromatic conductor from Figure 1; the Vase combines a dielectric BRDF and a dark greenish Lambertian base; the body of the Teapot is made of a colored conductor with an anisotropic halo component of vertical extent. All other materials do not use our composite BRDF model.
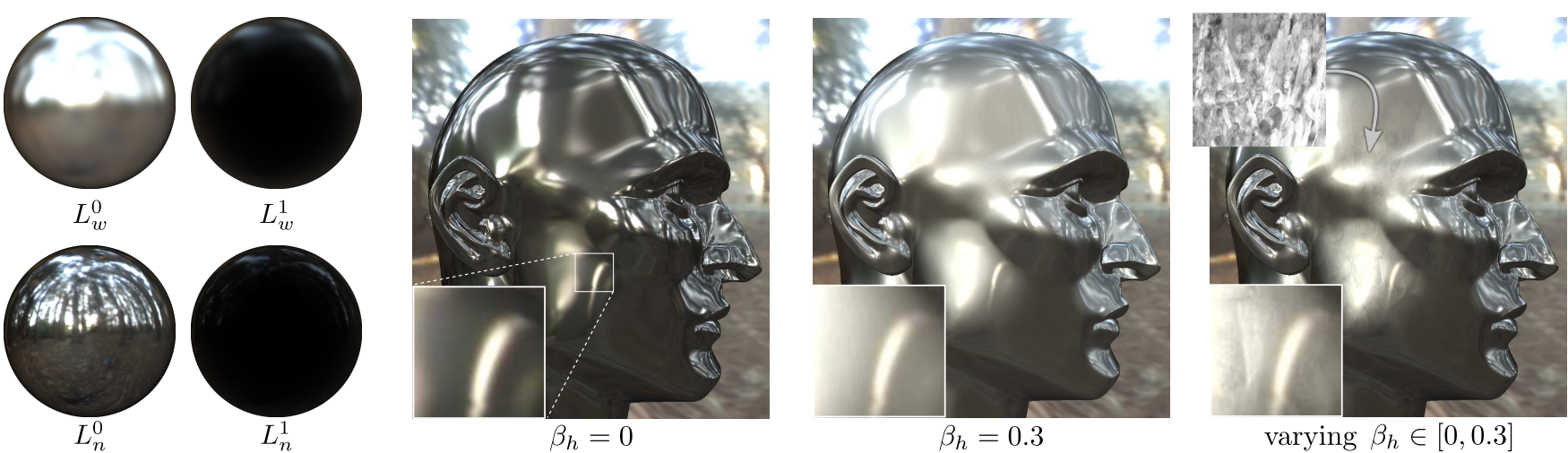

varying $\beta_{h} \in[0,0.3]$

Figure 12: For real-time rendering, we compute four pre-filtered environment maps $L_{n}^{0}, L_{n}^{1}, L_{w}^{0}$ and $L_{w}^{1}$, visualized on the four spheres on the left (we use $\alpha_{n}=0.012, \lambda_{h}=6.4$ ). As shown on the right, this permits the manipulation of haziness in real-time (we fix $r_{c}=0.5$ ), either for the whole object or locally using a texture map (finger prints applied to the side of the face). The supplemental video shows a live capture.

Inserting Equation 10 inside Equation 1 then 9 yields:

$$
L_{r}=(1-\beta)\left(L_{n}^{0} r+L_{n}^{1}\right)+\beta\left(L_{w}^{0} r+L_{w}^{1}\right),
$$

where $L_{\{n, w\}}^{0}$ and $L_{\{n, w\}}^{1}$ are reflected radiance functions obtained by replacing $f_{r}$ in Equation 9 with $f_{\{n, w\}}(1-\Delta)$ and $f_{\{n, w\}} \Delta$ respectively.
The main benefit of Equation 11 is that $L_{r}$ becomes a linear function controlled by physical parameters $\beta$ and $r$. Artist-controlled perceptual parameters may thus be converted to physical parameters using our haze mapping, and Equation 11 evaluated on the fly. This is shown in Figure 12 and the supplemental video, where we demonstrate interactive spatial variations of haziness. 
Another advantage of this approach is that it might be used to control hazy gloss at the compositing stage. The idea is to output render buffers for $L_{n}^{0}, L_{n}^{1}, L_{w}^{0}$ and $L_{w}^{1}$, as well as auxiliary buffers holding material parameters $\beta, r$ and $p$. The compositing artist then edits $\beta_{h}$ and $r_{c}$ obtained from auxiliary buffers, which are then interactively converted back to modified $\beta$ and $r$ buffers and plugged into Equation 11 to yield a new composite. Preliminary compositing results are shown in the supplemental document.

\section{Discussion and future work}

We have introduced a simple yet effective approach to the artistic control of hazy gloss for physically-based shading. The haze effect is controlled independently of the BRDF peak, in particular granting control over spatial variations of haze. Our method relies on a mapping from perceptual to physical parameters that only requires a few lines of code. Since it works with existing BRDF models, it may be easily integrated in existing rendering pipelines without much effort. Its simplicity is also key to its integration in real-time rendering applications or compositing pipelines.

In terms of performance, our haze mapping adds a negligible overhead compared to the evaluation of Equation 1. Using a composite model is obviously more costly compared to a singlecomponent specular BRDF. However, this is already used in production $\left[\mathrm{HMC}^{*}\right]$, which shows that the improved control over the shape of reflections is worth the increased computation time.

One effect of our haze mapping is to affect the global reflectivity $r$; hence it also indirectly modifies the refractive index $\eta+i \kappa$. We have seen in Figure 3 that in the case of dielectrics, the haziness $\beta_{h}$ should thus be restricted to limit $r$ to small values, and hence $\eta$ to physically-plausible indices. However, we prefer to leave this decision up to the artist, and only guarantee energy conservation $\left(\beta_{h}<\right.$ 1). Another consequence is that transmitted radiance is affected with increased haziness, as shown in Figure 13-left. In the case of conductors, $\beta_{h}$ need not be restricted; however, for very bright metals, the high core reflectivity $r_{c}$ does not leave much room for the halo component. There is no work-around this limitation since it is due to the energy conservation constraint. Our approach also inherits a limitation of Gulbrandsen's remapping [Gul14] for colored conductors. As the reflectivity is increased, the edge tint has less and less effect on material appearance: colors near occluding contours tend to become a blend between the edge tint and the reflectivity color. Since an increase in haziness results in an increase in global reflectivity, we obtain the same kind of color alteration near object boundaries as shown in Figure 13-right.

We have assumed throughout that the narrow and wide components $f_{n}$ and $f_{w}$ of Equation 1 are defined using the same type of BRDF function. One may want to use two different types of functions, but this would complicate the peak ratio formula $p$ without adding much more expressivity to the model. We have also limited our composite BRDF to a pair of components to be in accordance with existing perceptual studies on hazy gloss [VBF17]. Adding a third component would bring two additional degrees of freedom, but it is not clear how they might affect perception: would the new component be perceived independently or as part of the halo? As shown in the supplemental document, our preliminary tests favor

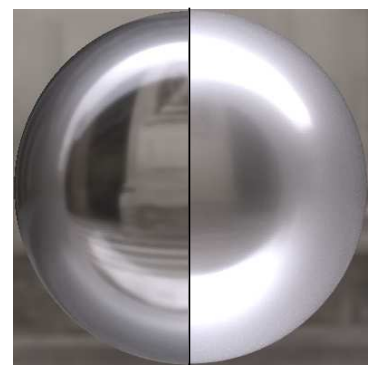

$\beta_{h}=0 \quad \beta_{h}=0.1$

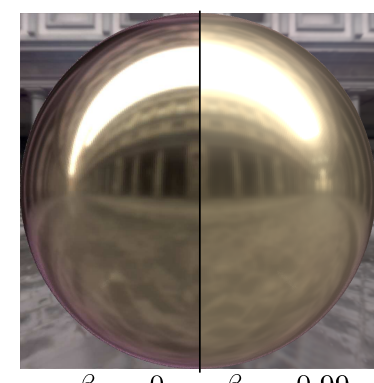

$\beta_{h}=0 \quad \beta_{h}=0.99$
Figure 13: Increasing haziness modifies the refractive index. Left: for a dielectric, transmitted radiance is affected as seen by comparing background distortions between the two halves (we use $\left.r_{c}=0.04\right)$. Right: for a conductor, edge tint is affected in the left half but is hardly visible in the right half $\left(\right.$ we use $\mathbf{r}_{c}=[0.6,0.52,0.3]$ and $\mathbf{g}=[1,0,0.85])$. In both cases, $\alpha_{n}=0.02$ and $\lambda_{h}=4$.

the latter interpretation; however, further perceptual experimentations are required to evaluate whether this is always the case.

One might wonder how our perceptual model performs on the fitting of measured BRDF data. Since our perceptual and physical models are connected by a bijection, the space of BRDFs they span is by definition the same; hence fitting (like rendering) can be performed using Equation 1. This has already been addressed in previous work [NDM05], where it is reported that using a pair of Cook-Torrance components reduces the fitting error by more than $25 \%$ for 26 out of 100 measured BRDFs of the MERL database.

Nevertheless, the only valid physical interpretation of our model is that of a mixture of distributions such as partially polished singlelayer materials. Our composite BRDF is thus not adapted to the modeling of bi-layered materials, as we assume a common Fresnel term for both narrow and wide components. Yet it would be interesting to provide control over hazy gloss in multi-layered materials through an inverse design approach. This represents a challenge as it is not clear how different layer configurations might affect perceived haziness. In addition, other perceptual dimensions are likely to emerge with more complex materials: in particular, some layers may be disentangled visually while others might not - a property we may call "layeredness". Finding explicit correspondences between physical and perceptual properties of multi-layered materials is an exciting direction of research for future work.

Acknowledgements We would like to thank Laurent Belcour and the anonymous reviewers for their feedback. This project has been supported by the ANR VIDA (ANR-17-CE23-0017). We are grateful to the Aim@shape library for 3D models and Paul Debevec for environment maps. The SpeedShape model is courtesy of Jiri Filip.

\section{References}

[AS00] Ashikhmin M., ShIRley P.: An anisotropic Phong BRDF model. Journal of Graphics Tools 5, 2 (2000), 25-32. 5, 6, 7

[AST97] ASTM: Standard Test Methods for Measurement of Gloss of High-Gloss Surfaces by Goniophotometry. Standard E430-97, American Society for Testing and Materials, West Conshohocken, PA, 1997. 2

[Bli77] BLINN J. F.: Models of light reflection for computer synthesized pictures. SIGGRAPH Comput. Graph. 11, 2 (July 1977), 192-198. 6, 7 
[BS63] BECKMANN P., SPIZZICHINO A.: The scattering of electromagnetic waves from rough surfaces. International series of monographs on electromagnetic waves. Macmillan, New York, 1963. 1

[BSH12] Bagher M. M., Soler C., Holzschuch N.: Accurate fitting of measured reflectances using a Shifted Gamma micro-facet distribution. Computer Graphics Forum 31, 4 (2012), 1509-1518. 1, 2, 7 , 8

[Bur12] BuRLEY B.: Physically based shading at Disney, 2012. 1, 2, 7,

[CT82] COOK R. L., TORRANCE K. E.: A reflectance model for computer graphics. ACM Transactions on Graphics 1, 1 (1982), 7-24. 1, 2, 7

[CTL89] Church E. L., TAKacs P. Z., Leonard T. A.: The prediction of BRDFs from surface profile measurements. In Scatter from $\mathrm{Op}$ tical Components, Proceedings of SPIE (1989), vol. 1165, pp. 136-150. 1,8

[Dis11] Disney: Brdf explorer. https://www. disneyanimation.com/technology/brdf.html, 2011

[Gul14] GULBRANDSEN O.: Artist friendly metallic fresnel. Journal of Computer Graphics Techniques (JCGT) 3, 4 (2014), 64-72. 3, 6, 11

[Hei14] HeItz E.: Understanding the masking-shadowing function in microfacet-based BRDFs. Journal of Computer Graphics Techniques 3 , 2 (2014), 48-107. 7

[HH87] Hunter R. S., HAROLD R. W.: The Measurement of Appearance, 2nd ed. Wiley, New York, NY, 1987. 2

[HMC*] Hill S., McAuley S., Conty A., Drobot M., Heitz E., Hery C., Kulla C., Lanz J., Ling J., Walster N., Xie F., MiCCIULLA A., VILLEMIN R.: Physically based shading in theory and practice. In ACM SIGGRAPH 2017 Courses. 2, 11

[HP17] Holzschuch N., PaCANOwski R.: A Two-Scale Microfacet Reflectance Model Combining Reflection and Diffraction. ACM Transactions on Graphics 36, 4 (Aug. 2017), 12. Article 66. 1

[JdJM14] JAKOB W., D'EON E., JAKOB O., MARSCHNER S.: A comprehensive framework for rendering layered materials. ACM Trans. Graph. 33, 4 (July 2014), 118:1-118:14. 1

[Kaj86] KAJiYA J. T.: The rendering equation. In Proceedings of the 13th Annual Conference on Computer Graphics and Interactive Techniques (New York, NY, USA, 1986), SIGGRAPH '86, ACM, pp. 143-150. 8

[Kar13] KARIS B.: Real Shading in Unreal Engine 4. Tech. rep., Epic Games, 2013. 9

[KVHSO0] Kautz J., VÁzQuez P.-P., Heidrich W., Seidel H.-P.: Unified approach to prefiltered environment maps. In Proceedings of the Eurographics Workshop on Rendering Techniques 2000 (London, UK, UK, 2000), Springer-Verlag, pp. 185-196. 9

[LFTG] Lafortune E. P. F., Foo S.-C., Torrance K. E., GreenBERG D. P.: Non-linear approximation of reflectance functions. In Proceedings of ACM SIGGRAPH 97. 2

[LKYU12] LÖW J., Kronander J., YNnERMAN A., Unger J.: Brdf models for accurate and efficient rendering of glossy surfaces. ACM Trans. Graph. 31, 1 (Feb. 2012), 9:1-9:14. 1, 2, 7, 8

[NDM05] NGan A., Durand F., MatusiK W.: Experimental analysis of BRDF models. In Rendering Techniques '05 (Proceedings of the 16th Eurographics Symposium on Rendering) (2005), Bala K., Dutré P., (Eds.), Eurographics, pp. 117-126. 2, 11

[NRH*77] Nicodemus E. F., Richmond C. J., Hsia J. J., GinsBERG W. I., LIMPERIS L. T.: Geometrical Considerations and Nomenclature for Reflectance. Tech. Rep. NBS MN-160, National Bureau of Standards, 10 1977. 2

[PFG00] Pellacini F., Ferwerda J. A., Greenberg D. P.: Toward a psychophysically-based light reflection model for image synthesis. In Proceedings of ACM SIGGRAPH 2000 (New York, NY, 2000), Akeley K., (Ed.), ACM, pp. 55-64. 2
[RBMS17] RIBARDIÈre M., BRINGIER B., MENEVEAUX D., SIMONOT L.: STD: Student's t-Distribution of Slopes for Microfacet Based BSDFs. Computer Graphics Forum 36, 2 (2017), 421-429. 1, $2,7,8$

[Rus98] RUSINKIEWICZ S. M.: A new change of variables for efficient BRDF representation. In Rendering Techniques ' 98 (Proceedings of the 9th Eurographics Workshop on Rendering) (1998), Drettakis G., Max N., (Eds.), Eurographics, pp. 11-22. 2

[Sch94] Schlick C.: An inexpensive brdf model for physically-based rendering. Computer Graphics Forum 13, 3 (1994), 233-246. 5, 9

[SGM*16] Serrano A., Gutierrez D., MyszKowski K., Seidel H.-P., MASIA B.: An intuitive control space for material appearance. ACM Trans. Graph. 35, 6 (Nov. 2016), 186:1-186:12. 2

[Smi67] SмIтH B.: Geometrical shadowing of a random rough surface. IEEE Trans. on Antennas and Propagation 15 (1967), 668-671. 7

[Sta99] STAM J.: Diffraction shaders. In Proceedings of the 26th Annual Conference on Computer Graphics and Interactive Techniques (New York, NY, USA, 1999), SIGGRAPH '99, ACM Press/Addison-Wesley Publishing Co., pp. 101-110. 1

[TR75] Trowbridge T. S., ReITZ K. P.: Average irregularity representation of a rough surface for ray reflection. J. Opt. Soc. Am. 65, 5 (May 1975), 531-536. 1

[VBF17] VANGORP P., BARLA P., Fleming R. W.: The perception of hazy gloss. Journal of Vision 17, 5 (2017), 19:1-17. 1, 2, 3, 4, 11

[War92] WARD G. J.: Measuring and modeling anisotropic reflection. Computer Graphics (Proceedings of ACM SIGGRAPH 92) 26, 2 (1992), 265-272. 2, 7

[WMLt07] Walter B., Marschner S. R., Li H., Torrance K. E. Microfacet models for refraction through rough surfaces. In Proceedings of the 18th Eurographics Conference on Rendering Techniques (2007), EGSR'07, Eurographics Association, pp. 195-206. 1, 7

[WW07] WEIDLICH A., WILKIE A.: Arbitrarily layered micro-facet surfaces. In Proceedings of the 5th International Conference on Computer Graphics and Interactive Techniques in Australia and Southeast Asia (New York, NY, USA, 2007), GRAPHITE '07, ACM, pp. 171-178. 1

\section{Appendix}

The quadratic rational Bézier curve $C$ with control points $\left\{\mathbf{p}_{0}, \mathbf{p}_{1}, \mathbf{p}_{2}\right\}$ and weights $\{1, w, 1\}$ of Figure 3 is given by:

$$
C(u)=\frac{(1-u)^{2} \mathbf{p}_{0}+2(1-u) u w \mathbf{p}_{1}+u^{2} \mathbf{p}_{2}}{(1-u)^{2}+2(1-u) u w+u^{2}},
$$

We first invert the first coordinate of $C$ to retrieve $u$, yielding:

$$
\begin{aligned}
u & =\frac{b-\sqrt{\Delta}}{2(b-1)}, \\
\Delta & =b^{2}-4(b-1) r_{c}, \\
b & =2\left(r_{c}(1-w)+w p\right) .
\end{aligned}
$$

This is the unique solution that yields a parametric coordinate in the desired $[0 . .1]$ range. To avoid numerical inacuracies when $b \approx 1$ we transition to a Taylor expansion of $u$ in a small window around the corresponding core reflectivity $r_{c}^{\star}$ :

$$
u \approx \frac{2 w p-1-\left(4 w^{2} p^{2}-4 w^{2} p+1\right)\left(r_{c}-r_{c}^{\star}\right)}{2(w-1)}
$$

with $r_{c}^{\star}=\frac{(1 / 2)-w p}{1-w}$. The halo intensity at $\theta_{d}=0$ is then directly obtained from the second coordinate of $C$, yielding:

$$
k_{h}=\frac{2(1-u) u w \beta_{h}}{(1-u)^{2}+2(1-u) u w+u^{2}} .
$$

